\title{
Analysis of Key Issues on Man-Control to System-Control Leap in Autonomous Driving
}

\author{
Zongwei Liu'1,2, Hong Tan ${ }^{1,2}$, Han Hao ${ }^{1,2}$, Fuquan Zhao ${ }^{1,2, a}$ \\ 1 State Key Laboratory of Automotive Safety and Energy, Tsinghua University, Beijing 100084, China \\ 2 Tsinghua Automotive Strategy Research Institute, Tsinghua University, Beijing 100084, China
}

\begin{abstract}
Autonomous driving is recognized as a global development direction and a major opportunity. The function and use of the vehicle has changed profoundly. The vehicle is gradually transformed from a simple transportation tool to a smart mobile space. The ultimate goal of autonomous driving is to achieve driverless driving. In the course of its development, man-control will gradually turn into to system-control. In other words, the transition from level 3 (L3) to level 4 (L4) is a fundamental leap. At present, the specific path to achieve this leap is not yet clear. Different companies have different and even opposite thinking and choices. In this study, the grading standard for autonomous driving was clearly explained, and the technical route selection of the company was analysed. Based on the analysis, the requirements of sensing, decision making, execution between the L3 and L4 were compared. Moreover, the key technical difficulties of L3 to L4 were clarified. In the end, suggestions on the commercialization of autonomous driving were given.
\end{abstract}

\section{Introduction}

A new round of scientific and technological revolution represented by cloud computing, big data, artificial intelligence is in the period of outbreak [1]. The technological revolution has brought about profound changes in the global automotive industry [2]. As the main core component of the traditional manufacturing industry, the automobile industry is entering an unprecedented period of change [3]. Traditional auto companies and technology companies are relying on multiple technology paths and multiple business models to achieve self-driving [4]. The functionality and application scenarios that automobiles can achieve are changing dramatically. Vehicles will gradually change from simple means of transportation to high-speed mobile office and living places, as well as information control terminals [5]. Driverless driving cars can achieve far richer functions and services than traditional cars.

Traditional car companies and technology companies are relying on a variety of technology paths, through a variety of business models to turn autonomous driving into reality. With kinds of advanced sensors, controllers and actuators, intelligent network automobile products with autonomous driving function have gradually entered the market [6]. In the practice of autonomous driving industry, some companies put forward the technical route of direct research and development of L4 products instead of L3 products. On the contrary, some auto companies still think it necessary to develop L3 products first. Different enterprises have very different thinking and choices. It's because the specific path to achieve the leap from L3 to L4 is not clear yet.

This paper is organized as follows. The next section describes the classification principle of autonomous driving and different choices of autonomous driving technology paths in current enterprises. Following that, technology paths between man-driven L3 and machinedriven L4 was compared. The subsequent section identified and analysed the key problems of leaping from L3 to L4. The final section provided suggestions on the commercialization of autonomous driving.

\section{Core Concepts and Key Technology Paths}

\subsection{Core concepts of autonomous driving}

The L3 is a key stage in the transition from man-control to system-control. According to the latest revision of SAE J3016(TM) by SAE International, autonomous driving is subdivided into six levels from L0 to L5 [7], as shown in Figure 1. The classification is based on the division of responsibility for performing steering and speed changes, the responsibility of monitoring the driving environment, the responsibility of dynamic driving task retreat and the driving system capability. Six levels correspond to the improvement of the degree of machine replacing man driving.

\footnotetext{
${ }^{a}$ Corresponding author: zhaofuquan@tsinghua.edu.cn
} 


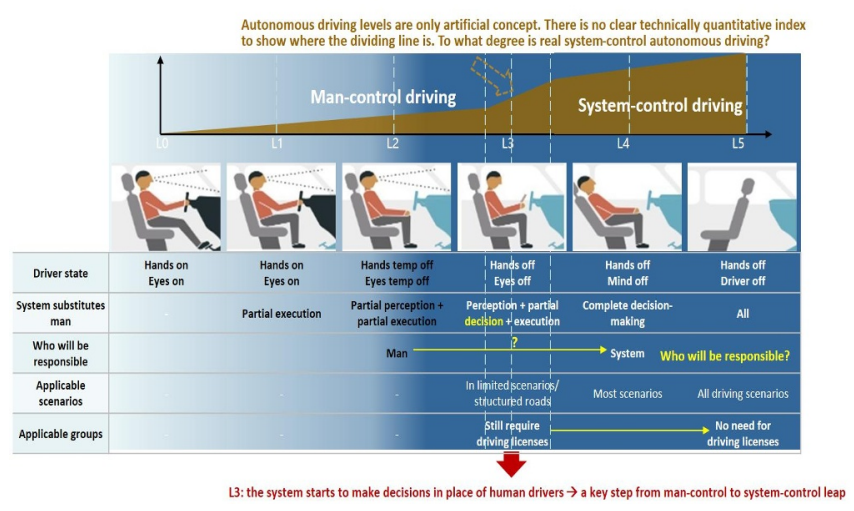

Figure 1. Classification principle of autonomous driving.

L3 self-driving allows human drivers to drive with their hands and eyes separated. But when the system makes a request, the human driver must respond appropriately. Machine replacements for human complete perception, execution and partial decision-making at the L3. However, the L4 autonomous driving can replace all decision making decisions and all driving operations. When the system issues a takeover request, the vehicle will ensure its own safety even if the human driver does not respond.

In fact, the classification is just a human concept. At which stage to achieve the transition from man-control to system-control, there is no recognized exact definition and quantitative technical indicators. As far as the definition of SAE is concerned, it is clear that the L3 level is a very critical step in achieving the leap. Because the machine has begun to replace people to make some decisions at this point. And the L4 level has already undoubtedly entered the stage of machine-led driving.

\subsection{Different technology paths choices for L3 and L4 autonomous driving for enterprises}

In the practice of autonomous driving, some companies have proposed to directly develop L4 products across L3. In contrast, other companies believe it is necessary to introduce L3 products first. For companies that choose to develop L4 directly, the considerations include user experience and security risks. From the perspective of user experience, some traditional car companies believe that L3 products have no commercial value. The reason is that the vehicle's irregular prompts and requests to take over the driving rights will cause confusion to the driver and even make it more tense than driving a traditional vehicle. Moreover, if the driver fails to take over within a limited time or can not take over the driving, L3 products will bring huge security risks. Indeed, this kind of unexpected situation cannot be completely avoided. In order to solve this problem fundamentally, L3 must be able to achieve a high level of driving. In other words, the autopilot does not send a request to the human driver in most cases. However, such L3 is very close to L4. Therefore, it is better to directly develop L4 products.

The company that advocates skipping L3 and heading straight to L4 is a radical. Its market advantage is that it is easier to get a larger share in emerging markets such as taxi services, automatic distribution services, and mobile travel services. Those who occupy market share first can give priority to building brand value, win consumer trust, and obtain possible natural monopoly. Its design and manufacturing advantages can be "one step in place". In another words, companies could focus on designing a centralized electronic control architecture for driverless driving at the beginning, instead of adding patches to the regional electronic control unit.

In contrast, most car companies represented by Audi still believe that L3 products have commercial value. It is believed that L3 products can solve user pain points under certain circumstances. For example, Audi has launched the L3 product. The A8L can achieve autonomous driving in scenarios to ensure safety such as low speed $(60 \mathrm{~km} / \mathrm{h}$ or less) and less dangerous urban congestion roads. At the same time, sufficient time to take over is reserved on the interaction scheme. Use stepby-step alerts to optimize user experience. Considering that the recent launch of L4 products is very difficult, car companies make this technical route choice. Not only does it take time to do technical iterations and cost reductions, but there are also issues of consumer trust and acceptance. However, in order to seize market opportunities as soon as possible, the introduction of L3 products has become a good choice to increase the selling point of products.

The company that advocates a gradual evolution from L3 to L4 are conservative. The technological advantage is that the number of sensors required for L3 products is small. In addition, L3 products have low requirements for sensor performance, software complexity, computing power, redundant design and infrastructure. The presence of L3 also gives human drivers the opportunity to become familiar with handing over driving tasks to the system. However, the technical route pays more attention to the reliability of the technology. The technology should enable the automated driving system to handle any situation within 5 to 10 seconds necessary for the human driver to be aware of the need to take over and control. Because if a certain accident is caused, the negative public opinion will offset the gain brought by L3. More seriously, it also delays the time when L4 products enter the market.

In fact, the choice of two technology paths is reasonable. Specifically, whether to develop L4 directly or launch L3 first depends not only on the orientation and planning of the enterprise for its own development, but also on the essential difference between L3 and L4. More importantly, enterprises should have a comprehensive and accurate understanding of key issues on man-control to system-control.

\section{Comparison and leaping difficulties}

\subsection{Comparison of L3 and L4 autonomous driving}

Autonomous driving technology includes perception, decision-making and execution. Although there are some 
similarities or inheritances in these three aspects, there are still quite different between L3 and L4, as shown in Figure 2.

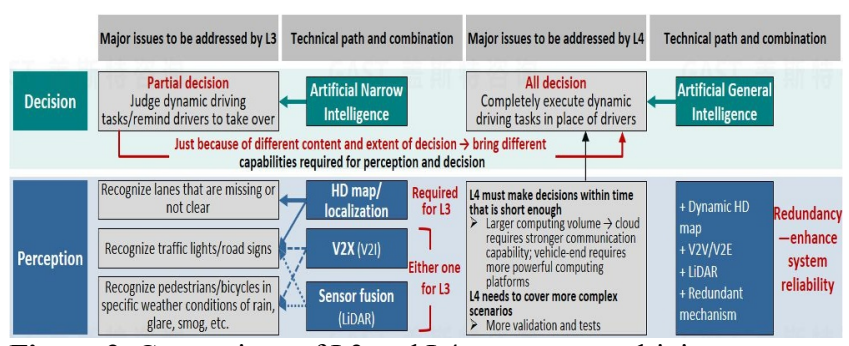

Figure 2. Comparison of L3 and L4 autonomous driving

From the perspective of decision making, applying general artificial intelligence to L3 can be sufficient to meet the needs of partial decision-making, including judging dynamic driving tasks and alerting human driver to take over. In contrast, L4 requires the application of strong artificial intelligence to completely replace people for all decisions and perform dynamic driving tasks. Due to the different content and degree of decision-making, the corresponding capabilities required for perception and decision-making are completely different.

From a perceptual perspective, L3 uses high-precision maps and positioning to identify missing and blurred lane lines. Identify traffic lights and road signs via V2X (car network) [8] or sensor fusion. And identifying pedestrians and bicycles under certain conditions such as rain, glare, and haze [9], [10]. For L4, dynamic highprecision maps, V2X and sensor fusion including Lidar are indispensable. Ensure that decisions can be made in a short enough time and cover more complex scenarios. In addition, redundant mechanisms are needed to enhance the reliability of the system.

From the perspective of implementation, the technical route of L3 and L4 autonomous driving is basically the same. Linear control of actuator is a necessary condition for autonomous driving above L3. On this basis, L4 enlarges the redundancy of actuator.

The choice of two technology paths is to develop L4 directly or release L3 first. The core of the controversy is whether L4 can be developed gradually through the accumulation and promotion of L3 technology. Based on the above analysis, L3 and L4 are not simple progressive relationships. This is not only a qualitative leap in technology, but also a quantitative leap in workload. In addition, it involves many issues outside the technology. Therefore, the premise of achieving L4 is to achieve a fundamental breakthrough. However, the development of L3 can indeed support L4 in some aspects, including the accumulation of common technologies such as sensors and algorithms.

\subsection{Key difficulties in leaping from L3 to L4}

\subsection{1 decision-making}

At present, the sensing and execution technological route of autonomous driving has been relatively clear, but the reliable decision-making technological route is still inconclusive. As environmental complexity and task complexity continue to increase, the decision-making techniques required for autonomous driving must also be upgraded. The environment goes from a controlled environment to a regular environment, and finally to an unpredictable environment. The driving task changes from the pre-set destination to the predicted destination, and finally to the destination dynamically. Environmental complexity and task complexity are gradually increasing. The decision-making techniques required for autonomous driving must also be upgraded. Decision-making techniques should respond to change from established programming methods based on rules to machine learning based on scene and conditional probabilities, and finally to deep learning based on neural networks with automatic learning capabilities. In other words, the transition from L3 to L4 needs to adapt to more complex tasks and environments, and the decision-making difficulty varies greatly.

Decision-making of L4 requires deep learning. There are two main types of deep learning methods currently applicable to autonomous driving, namely semantic abstraction and end-to-end learning. Semantic abstraction is more common in the choice of automotive companies. he principle is to decompose the automatic driving problem into several modules such as detecting vehicles, lanes, obstacles. And then expert expertise and experience are used to tag and annotate your data to train each module. The advantage of this method is the low rate of failure. Once the error occurs, the source can be traced back and the unknown situation of the burst can be handled. The disadvantage is that a lot of up-front preparation and programming is required during the training process. And a large amount of redundant data marks are generated.

And end-to-end deep learning is more concerned by IT companies. The principle is to directly learn the real driving data of human beings. The system is equivalent to a black box of input perception and output decision. The advantage of this method is that it does not require precise marking and annotation of scene data during training., and the system as a whole for self-learning and optimization. As training data increases, the system becomes more reliable. The disadvantage is that a higher level of artificial intelligence engineers are required to train the system with a larger amount of data. In essence, both of these deep learning methods are highly dependent on big data, and getting full and complete big data is far more difficult than imagined.

\subsection{2 intuition}

Human drivers can intuitively predict the behavior of other road traffic participants while driving. This plays an important role in the safety of drivers and others. However, it is very difficult for intelligent vehicles to understand human intentions and predict human behavior. This is also one of the fundamental challenges facing machine-driven driving. There is no recognized solution for this problem in the industry. There are three possible cracking methods. The first is to let the machine 
thoroughly understand human behavior and intuition, through in-depth research in brain science, behavioral science, and cognitive science. The second is to completely remove the human driver from the road traffic system. The focus of this problem is changed to the prejudgment behavior of intelligent vehicles. The third is to let the machine "learn" the intuition through various algorithm upgrades, so that the machine is infinitely close to people. Obviously, these three paths are difficult to achieve in the short term.

\subsubsection{Testing and verification}

At present, the basic idea of using artificial intelligence technology to solve the problem of autonomous driving is relying on massive data. In order to solve problems accurately and effectively, it is necessary to ensure the completeness of the collected big data. The study pointed out that if the human driver's driving safety data is used as a reference, in order to prove that the active safety performance of the self-driving car exceeds man-control, the required test mileage may be as high as 5-6 million kilometres [11]. In addition, traditional vehicles only need to test the reliability of vehicle control and actuators, while intelligent vehicles also need to test the ability of perception, decision-making and related hardware and software. Correspondingly, it brings some new problems. For example, accidents that have not been recorded in the history of man-control also need to be avoided by autonomous driving vehicles. How to collect such scene data comprehensively? Another example is that intelligent vehicles may cause new traffic accidents. How to accumulate and verify such scenes? In addition, it is also necessary to consider how people's driving behaviour will change in the environment where people and machines work together.

The only way to solve the problem is to continuously increase the accuracy and proportion of virtual testing through scientific methods based on closed environment and open road measured data [12]. But the virtual test model does inevitably simplify the real situation. And the verification of its effectiveness is very difficult. Obviously, there is still a long way to go.

In addition to the above three major difficulties, autonomous driving has problems other than technology to face, including the problems about human trust in machine-driven vehicles, social ethical issues in which machines replace people making decisions [13], and laws and regulations that are contrary to current regulations. In the long run, these social problems will eventually be resolved with changes in consumer concepts, advances in technology, and improvements in regulations. But this takes time and must be a long-term process. For companies, the judgment of this time process is closely related to product and technology planning.

\section{Suggestions on the commercialization}

First of all, the fact is that L4 products that meet the needs of various scenarios do not have the possibility of mature mass production in the short term. Because L4 is fundamentally different from L3 in terms of development difficulty, technical cost, subject responsibility and social issues. In addition, there are still many problems that cannot be solved simply by relying on the car company. In other words, the most likely automatic production in the near future is the L3 product. Recently, some companies have expressed that they have been too optimistic about the progress of the autonomous driving development process. Therefore, if the company only layouts and develops L4 products, it is likely to lose the product selling point that is recently needed. Even enterprises will face the risk of mass production in the foreseeable future. Even if choosing the technical route to directly develop L4, companies should also develop L3 at the same time. This is not only a backup guarantee that the time for launching L4 products may lag, but many technical developments of L3 can also provide effective support for L4. In general, for companies with relatively limited strength and resources, it is more important to cut into L3 to achieve short-term gains, regarding the L4 technology as a long-term technical reserve.

Secondly, people do not need to be completely liberated during the driving process in the current stage. If the autonomous driving can effectively liberate the driver in certain scenarios for daily use, there is a good market prospect. Of course, the premise is to make sure the experience is good enough. For example, in a closed environment such as automatic parking in a parking lot, highway driving, and low-speed urban congestion conditions, L3 products are sufficient to meet consumer demand. This brings great convenience to consumers. Of course, the premise is that companies must make their L3 products good enough. In order to ensure the safety of man-machine switching during driving, it is necessary to make it as close as possible to L4. The bad driving experience caused by frequent requests for the driver to take over the vehicle should be considered. In this sense, there is still a lot of work to do for L3. In addition to continuing to increase investment in technology research, companies should also evaluate from the two dimensions of autonomous driving grading and driving scene grading. Enterprises should comprehensively analyze and systematically plan application scenarios suitable for different levels of autonomous driving products. Based on the difficulty of implementation, the expected industrialization time note for enterprises should be given.

Finally, in order to achieve sustainable development, enterprises must be based on the present and the future. For this reason, they must always be prepared. For autonomous driving, companies must balance the research reserve of technology with the mass production application of the product, and actively explore potential market opportunities corresponding to technology, as well as try innovative business model. In this sense, companies can completely customize the connotation of L3 and L4 products, and accelerate the promotion of autopilot products under common scenarios. At the same time, the current stage of car companies must consider the research reserve of L4 autonomous driving technology. One thing to emphasize is that the development of L4 should be effective in feeding back L3 autonomous driving technology products, rather than 
letting L4 technology and products develop in isolation. In specific practice, car companies should focus on strengthening cooperation and reserves in artificial intelligence, continuously accumulate and improve the database of autonomous driving application scenarios, and pay attention to and promote the development of new testing and verification methods. In specific practice, car companies should focus on strengthening cooperation and reserves in artificial intelligence. At the same time, the autonomous driving application scenario database needs to be continuously accumulated and improved. In addition, companies should pay attention to and promote the development of new testing and verification methods. Only in this way can the enterprise be invincible in the historical leap from man-control to system-control.

\section{Conclusive remarks}

L3 is the technology watershed in the process of enterprise achieving driverless driving. L3 level is a very critical step in achieving the leap. Because the machine has begun to replace people to make some decisions at this point.

From the perspective of technical realization of autonomous vehicles, L3 and L4 are not simple progressive relationships. This is not only a qualitative leap in technology, but also a quantitative leap in workload. The development of L3 can indeed support L4 in some aspects, including the accumulation of common technologies such as sensors and algorithms. The three technical difficulties that span from L3 to L4 are decision making, intuition, and testing and verification. The main difficulty in decision making is that getting full and complete big data is far more difficult than imagined. But, the decision systems of L4 based on deep learning are highly dependent on well-completed big data. And, the difficulty in intuition is that it is very difficult for autonomous vehicles to understand other human' $s$ intention and predict their behaviour. In addition, the reliability of autonomous vehicles is difficult to verify through real-world road testing and virtual world simulations.

From the perspective of the commercialization of autonomous driving, not only does it take time to do technical iterations and cost reductions, but there are also issues of consumer trust and acceptance. If the L3 product can effectively liberate the driver in certain scenarios for daily use, there is a good market prospect. Enterprises should comprehensively analyze and systematically plan application scenarios suitable for different levels of autonomous driving products. At the same time, the current stage of car companies must consider L4 autonomous driving technology research reserve.

\section{Acknowledge}

This research is supported by the National Natural Science Foundation of China (U1764265). The authors would like to thank all partners within for their cooperation and valuable contribution.

\section{References}

1. X. Miao. Smart Factory and the Transformation \& amp; Upgrading of Equipment Manufacturing Industry. Process Automation Instrumentation. 35(03), 1-6 (2014)

2. F. Zhao, Z. Liu. Strategy direction of building powerful automotive industry in China. Auto Industry Research, (10), 4-7 (2014)

3. Z. Liu. Fuquan Zhao's Insights on Automotive Industry [M]. Beijing: China Machine Press, 57-63 (2017)

4. F. Zhao, Z. Liu., H. Hao, T. Shi. Characteristics, trends and opportunities in changing automotive industry. Journal of Automotive Safety and Energy, 9(3), 233-249 (2018)

5. X. Kuang, F. Zhao, H. Hao, Z. Liu. Intelligent connected vehicles: the industrial practices and impacts on automotive value-chains in China. Asia Pacific Business Review, 24(1), 1-21 (2018)

6. K. Li, Y. Dai, S. Li, M, Bian. State-of-the-art and technical trends of intelligent and connected vehicles. Journal of Automotive Safety and Energy, 8(1), 1-14, (2017)

7. SAE J3016(TM) Taxonomy and Definitions for Terms Related to Driving Automation Systems for On-Road Motor Vehicles [s]. SAE International,1819 (2018)

8. S. Chen, J. Hu, Y. Shi. LTE-V: A TD-LTE based V2X solution for future vehicular network. IEEE Internet of Things Journal, 3(6),997-1005 (2016)

9. C. Ilas. Electronic sensing technologies for autonomous ground vehicles: A review. $20138^{\text {th }}$ International Symposium on Advanced Topics in Electrical Engineering (ATTE), IEEE, 1-6 (2013)

10. J. Varghese, R. Boone. Overview of Autonomous Vehicle Sensors and Systems. International Conference on Operations Excellence and Service Engineering, 10-11 (2015)

11. N. Kalra, S. Paddock. Driving to safety: How many miles of driving would it take to demonstrate autonomous vehicle reliability? Transportation Research Part A: Policy and Practice, 94, 182-193 (2016)

12. B. Kim, Y. Kashiba, S. Dai. Testing Autonomous Vehicle Software in the Virtual Prototyping Environment. IEEE Embedded Systems Letters, 9(1), 5-8(2017)

13. P. Lin. Why ethics matters for autonomous cars[M]. Springer, 69-85(2016) 